\title{
A review of computational intelligence methods for traffic management systems
}

\author{
Vladimir llin ${ }^{a^{*}}$, Dragan Simića \\ a University of Novi Sad, Faculty of Technical Sciences, Trg Dositeja Obradovića 6, 21000 Novi Sad, Serbia
}

\begin{tabular}{|c|c|}
\hline ARTICLE INFO & A B S TRACT \\
\hline $\begin{array}{l}\text { DOI: } 10.31075 / \text { PIS.67.04.05, } \\
\text { Professional paper } \\
\text { Received: } \\
\text { Accepted: } \\
\text { Corresponding author: } \\
\text { v.lin@uns.ac.rs } \\
\text { Keywords: } \\
\text { Computational intelligence methods, } \\
\text { Neural networks, Fuzzy logic } \\
\text { systems, Evolutionary computation, } \\
\text { Traffic management systems } \\
\text { This article has been presented at the 8th } \\
\text { International Conference } \\
\text { "Towards a Humane City" } \\
11-12 \text { November 2021, N. Sad, Serbia }\end{array}$ & $\begin{array}{l}\text { One of the most important challenges in modern city life is to enable effective } \\
\text { and efficient traffic management system. Recently, computational intelligence } \\
\text { methods have become increasingly popular for traffic management system } \\
\text { design, application, and monitoring. Computational intelligence methods are } \\
\text { often deployed for managing traffic, that is for reducing mileage, congestion, the } \\
\text { use of fuels, and environmental impact. The aim of this paper is twofold. First, to } \\
\text { present the three main areas in a computational intelligence approach, namely } \\
\text { neural networks, fuzzy logic systems, and evolutionary computation. Second, to } \\
\text { emphasize their impact on various traffic management domains, including traffic } \\
\text { flow forecasting, traffic light control, traffic fatalities prediction, traffic sign } \\
\text { detection, and optimization of transportation networks. }\end{array}$ \\
\hline
\end{tabular}

\section{Introduction}

Due to the constant increase in the degree of motorization and individual motor traffic, cities are exposed to growing traffic problems and hence there is an increased need for the well-organized traffic management system. Traffic management can be defined as the organization, arrangement, guidance, and control of both stationary and moving traffic, including pedestrians, bicyclists and all types of vehicles (Underwood, 1990). Nowadays, to achieve efficient and effective traffic management system the use of information and communication technologies (ICT) and intelligent transportation systems (ITS) is required.

ITS are often deployed for collecting and interpreting traffic data in order to improve traffic flows. As an example, Internet of Things (IoT) devices can be used for collecting and analyzing the traffic data. However, in order to manage traffic data, that is to transform raw data on the input of an information system towards meaningful information on the output a middleware is required. For that purpose, various nature-inspired algorithms are implemented as part of software solutions that acts as a bridge between input and output in the ITS specialized for various traffic management domains.
In general, the use of algorithms for traffic management systems has multiple advantages for the organization, arrangement, guidance, and control of both stationary and moving traffic. These advantages include decrease of mileage, decrease of traffic congestion, decrease of the use of fuels, decrease of noise, etc. In that manner, computational intelligence methods have become increasingly popular for traffic management system design, application, and monitoring. Computational intelligence methods belongs to the class of nature-inspired techniques used to solve complex and multi-criteria problems in real-world environments. In traffic and transportation environment, nature-inspired techniques are often deployed for managing traffic and reducing congestion. Here we adopt the classification that differs three main areas in a computational intelligence approach, namely (1) neural networks, (2) fuzzy logic systems, and (3) evolutionary computation algorithms. Neural networks are inspired from human brain modeling and they present a trainable system along with pattern recognition abilities; fuzzy logic systems involve techniques and methods for reasoning under imprecision or uncertainty conditions, while evolutionary computation presents a type of stochastic optimization search and consists of swarm intelligence and evolutionary algorithms (Karaboga et al., 2014). 
The computational intelligence paradigm is often compared with artificial intelligence paradigm, soft computing techniques, and natural computing techniques (Figure 1). However, all paradigms use different methodologies to describe the main principles of their approaches.

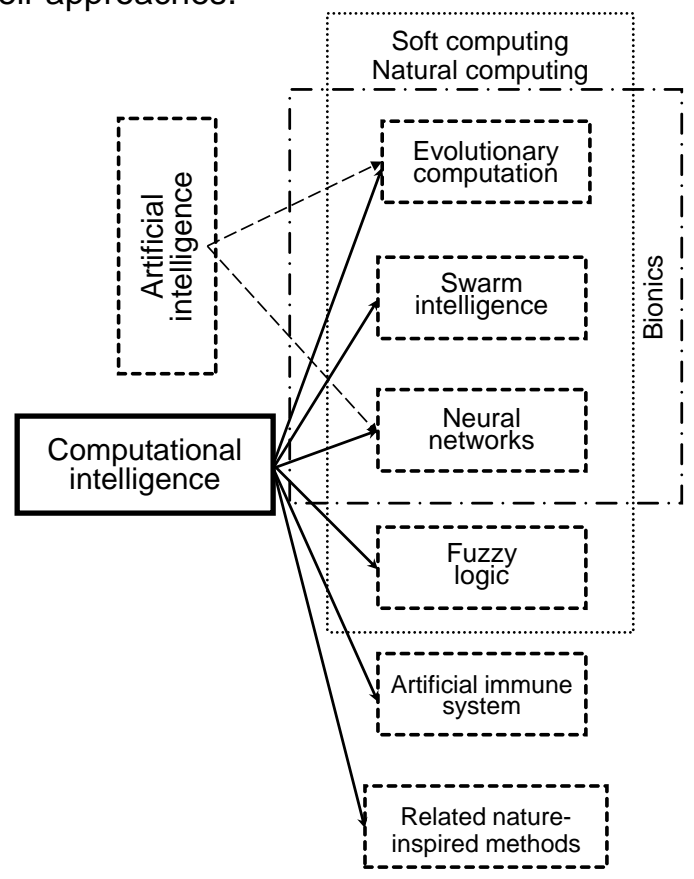

Figure 1. Computational intelligence methods and other computing techniques

Source: (Hanne \& Dornberger, 2017)

The rest of the paper is organized as follows. First, various applications of neural networks for traffic management systems are presented. After that, interactions between fuzzy logic systems and traffic management systems are discussed. Finally, evolutionary computation is introduced and some frequently used methods for traffic management systems are explained. The paper concludes with a discussion of the key findings and implications for future research.

\section{Neural networks and traffic management systems}

An artificial neural network (ANN) is a technique that mimics the function of biological neurons of brain as well as their interconnections. ANN belongs to the class of nonparametric technique, together with support vector machines, K-nearest neighbors' regression, etc. The ANN ability to reproduce nonlinear relationships between variables describing object's behavior and the ability to develop effective configurations is conducive to traffic management applications (Brzozowska, 2019).

An example of the simple ANN architecture is shown in Figure 2. The presented architecture includes network inputs, network outputs, input layer, hidden layer, output layer, and neurons.
Each neuron on the input calculates a sum of all weights included in the bias, while each neuron on the output comprises an activation function of a sum of all weights on input. In Figure 2, X represents the inputs, $W$ represents the corresponding weights for each input, $b$ represents the bias, and $Y$ represents the output. To calculate $\mathrm{Y}$, the ANN model applies an activation function to the inputs and bias.

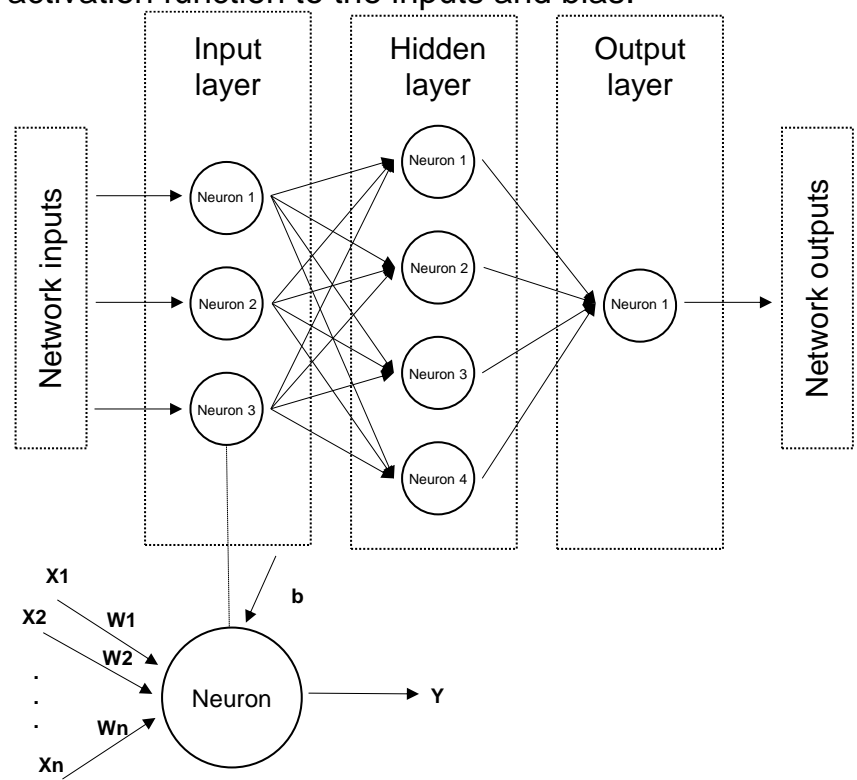

Figure 2. An example of artificial neural network with one hidden layer

ANN are often used for traffic prediction, which is an important aspect in all ITS models. Traffic prediction refers to the possibility to forecast future state of traffic variables, such as flow or speed based on historical or captured traffic data. Traffic prediction usually encompasses numerous characteristics of the traffic, such as different measurement intervals and time scale of prediction. In the literature, most of the ANN models used for traffic prediction fall into one of the following types: multilayer feedforward neural networks (MLFNN), time-delay neural networks (TDNN), recurrent neural networks (RNN), convolution neural networks $(\mathrm{CNN})$, deep belief networks (DBN), radial basis function neural networks (RBFNN), wavelet neural networks (WNN), and fuzzy neural networks (FNN) (Do et al., 2019). Of these methods, CNN are most frequently applied for traffic management systems. For example, CNN are used for recognizing highway traffic congestion in surveillance camera images, for intelligent traffic management for emergency vehicles, and for real time automatic urban traffic management (Cui et al., 2020; Meicler et al., 2020). Images generated by highway surveillance cameras usually have much more extensive range of perspective and thus larger area of background, including rich variance of scenes and road configurations (Cui et al., 2020). Therefore, CNN have a task to classify such images, without any special previous processing such as segmentation of objective roads. 
In the case of traffic management for emergency vehicles CNN model is deployed to accept the video from the traffic road and take quick decision to allow the emergency vehicles. Automatic urban traffic management includes monitoring and control of the traffic environment. The system is mostly designed for large cities in which traffic congestions need to be monitored and regulated. In (Meicler et al., 2020), authors propose CNN to classify non-motorized traffic participants (pedestrians, bikes, and kick scooters) and motorized vehicles (light vehicles, trucks, and motorbikes). In some cases, ANN are combined with some other methods to increase efficiency of the hybridized model. For example, deep neural networks are hybridized with bat algorithm (BA) to provide a dynamic form of traffic control in vehicular ad-hoc networks (Kannan et al., 2021). The proposed model has shown precise traffic flow predictions in real-time conditions and, as a result, the main traffic parameters are improved, including decrease in delay, fuel consumption, and emissions.

\section{Fuzzy logic systems and traffic management systems}

A fuzzy logic system (FLS) is a technique suitable to represent incomplete data and uncertain information by fuzzy sets instead of crisp set theory. An important advantage of FLS is to include expert's knowledge in their design which can be decisive feature for traffic management systems, e.g., in managing vehicles routes, traffic signals, creating new traffic management systems, etc. (Engelbrecht et al., 2007).

An example of the FLS architecture is shown in Figure 3. First phase, fuzzification, implies association of numerical values to fuzzy sets. An example of fuzzy set in traffic management domain can be low, medium, and high traffic flow. In the second phase, the logic of the fuzzy system is define by if-then rules in fuzzy inference. Membership functions are used to indicate the degree of dependency to each fuzzy set. Finally, the third phase, defuzzification, implies transformation of fuzzy sets to numerical values.

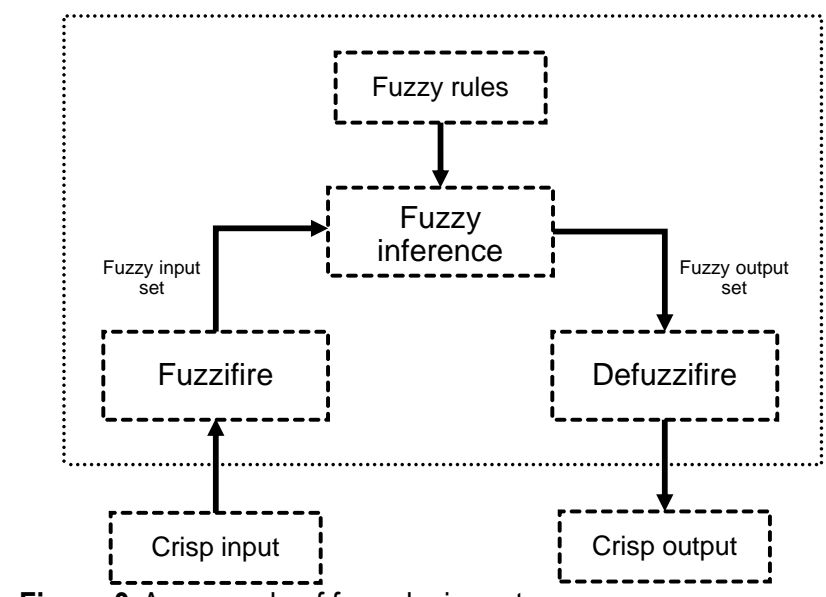

Figure 3 . An example of fuzzy logic system
With changing demand and traffic conditions over time, dynamic management is a requirement (Khattak \& Fontaine, 2020), that is real-time traffic management. One example of real-time traffic management is the use of autonomous vehicles equipped with various sensors and decision-making systems that enable increased traffic safety and decreased negative impact on the environment. Another example is allocation of sensors throughout the road network. There are usually obstacles to deploy sensors on the whole road network and in such situation new technologies, particularly FLS, can be used to estimate the missing information. Devaki et al. (2021) used four main criteria, namely economic, public and political, environmental, and traffic safety, and 13 sub-criteria using fuzzy multi-criteria decision making to prioritize the advantages of six different real-time traffic management methods, which are dynamic speed limits, lane control systems, variable message signs, ramp metering, traffic diversion, and integrating autonomous vehicles into other traffic management systems. Their results imply that the real-time traffic control integration with autonomous vehicles has the highest priority, while variable massage signs as a traffic management method is the least advantageous. FLS can also be used to propose new traffic management systems. In (Raskar \& Nema, 2021), a smart barricade system is proposed. This smart system is intended to localize nature-based barriers and barriers based on the intensity of traffic. The FLS is used to decide where to place the barriers and an algorithm, called, Modified Grasshopper Optimization Algorithm is used to find optimal membership limits. Besides for road traffic management, FLS is used for creating new railway traffic management systems (Blagojević et al., 2021) and air traffic management systems (Wei-jun et al., 2019).

\section{Evolutionary computation and traffic management systems}

Evolutionary computation (EC) is a technique that mimics phenomena from nature, such as natural evolution of life. EC belongs to the class of heuristic searching mechanism to find optimal or near-optimal solutions in different optimization problems. These techniques are characterized with population-based approaches which are highly adaptive for various applications (Figure 4). 


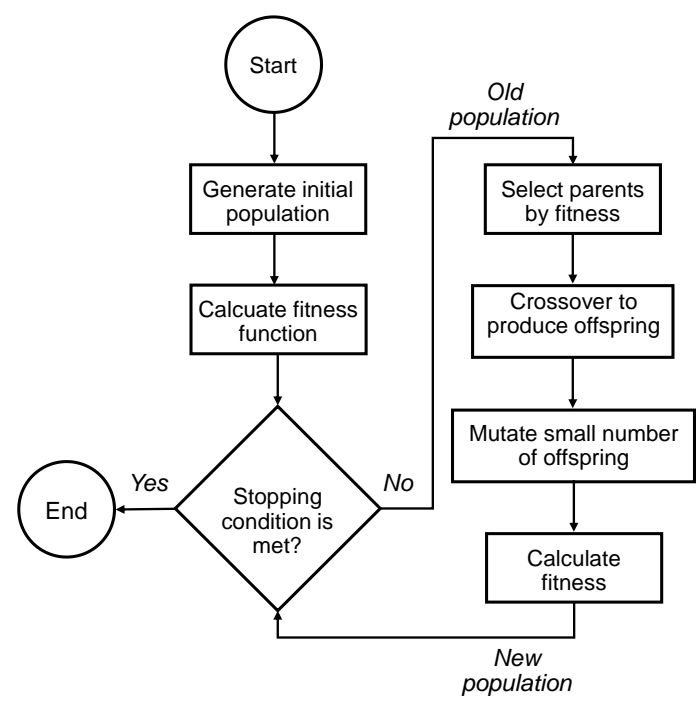

Figure 4. A general scheme for evolutionary algorithms Source: (Wilde et al., 2020)

In traffic management, EC has been often deployed for managing traffic and dealing with routing and scheduling problems. EC algorithms include evolutionary algorithms (genetic algorithms (GA), genetic programming (GP), learning classifier system (LCS), and so on), swarm intelligence (ant colony optimization (ACO), artificial bee colony (ABC), particle swarm optimization (PSO), and so on), and hybrid algorithms (Hu et al., 2015). Among them, GA, ACO, $A B C$, and $P S O$ are widely used for dealing with traffic management problems. Pseudocodes for GA, ACO, $\mathrm{ABC}$, and PSO are presented in Table 1.

Table 1. Pseudocodes for $G A, A C O, A B C$, and PSO

\begin{tabular}{|l|}
\hline \multicolumn{1}{|c|}{ GA } \\
\hline Initialization phase; \\
while stopping criterion is not satisfied \\
Evaluate population (fitness function); \\
Select best individuals for reproduction; \\
Perform crossover and create new pop;; \\
Apply the mutation operator; \\
Replace old population with new; \\
end while \\
\hline \\
\hline Initialization phase; \\
while stopping criterion is not satisfied \\
Construct ant solution; \\
Apply local search; \\
Update Pheromones; \\
end while \\
\hline \\
\hline Initialization phase; \\
while stopping criterion is not satisfied \\
Phase of employed bees; \\
Phase of onlooker bees; \\
Phase of scout bees; \\
Memorize the best solution; \\
end while \\
\hline Initialization phase; \\
while stopping criterion is not satisfied \\
Evaluation phase; \\
Update phase: \\
Update velocities; \\
end while \\
\hline \multicolumn{1}{c|}{ PSO solion; } \\
\hline
\end{tabular}

The GA is a metaheuristic search algorithm based on the biological evolutionary process of "the survival of the fittest" - the principle proposed by Charles Darwin. The purpose of using a GA is to find the individual from the search space with the best "genetic material". The quality of an individual is measured with a fitness function and the part of the search space to be examined is called the population. In traffic management, GA is deployed for optimizing traffic signals (Al-Madi \& Hnaif, 2021), prediction of severity of traffic accidents (Ospina-Mateus et al., 2021), and travelling salesman problem (llin et al., 2020).

The $A B C$ is a metaheuristic search algorithm inspired by the foraging habits of bees. The colony of artificial bees in $A B C$ contains three groups of bees: employed bees associated with specific food sources, onlooker bees watching the dance of employed bees within the hive to choose a food source, and scout bees searching for food sources randomly. In $A B C$, the position of a food source represents a possible solution to the problem and the nectar amount of a food source corresponds to the quality (fitness) of the associated solution. In traffic management, $A B C$ is deployed for traffic sign detection (Banharnsakun, 2018), traffic signal timings (Dell'Orco et al., 2014), and vehicle routing problem ( $\mathrm{Ng}$ et al., 2017).

The ACO is a metaheuristic search algorithm inspired by the foraging behavior of ants, which use pheromones as a communication medium to determine the best path from the food source to their nest. The pheromone trails are represented with numerical values and during the search those numerical values are adjusted. In traffic management, ACO is deployed for dynamic traffic routing and signalfree intersection traffic control for connected vehicles (Nguyen \& Jung, 2021), intelligent traffic control systems (Kumar et al., 2018), and traffic lights optimization (Elgarej et al., 2016).

The PSO is a metaheuristic search algorithm that simulates swarm of particles. The basic idea of the PSO algorithm is to mimics social behavior of bird flocks and fish schools searching for food. In comparison to previously mentioned methods, the PSO is relatively easy to implement, it has a less tendency to be trapped in a local optimum, and it requires less memory and CPU time. In traffic management, PSO is deployed for traffic flow forecasting (Zhang \& Wang, 2018), traffic light optimization ( $\mathrm{Hu}$ et al., 2018), and traffic fatalities prediction (Gu et al., 2018). 


\section{Conclusion and future work}

In this paper we have emphasized the importance of computational intelligence methods for traffic management design, application, and monitoring. The three main areas in a computational intelligence approach, namely neural networks, fuzzy logic systems, and evolutionary computation are explained and their applications in various traffic management domains are presented. Among them, evolutionary computation algorithms are explained in more detail and their applications in traffic flow forecasting, traffic light control, traffic fatalities prediction, traffic sign detection, and different optimizations of transportation networks are highlighted.

Future work could focus on extending the research on other computational intelligence areas, such as reinforcement learning or Q-learning. Potential research could also include the thorough analysis of one selected computational intelligence method and investigation on its applications in various traffic management domains. Likewise, the researchers could choose one traffic management domain and investigate which computational intelligence methods are the most adequate to be applied. Various applications of computational intelligence methods in logistics and supply chain management, such as transportation problems, inventory planning, scheduling, and location planning, can also be investigated.

\section{Acknowledgements}

This research has been supported by the Ministry of Education, Science and Technological Development through the project no. 451-03-9/2021-14/200156: "Innovative scientific and artistic research from the FTS (activity) domain".

\section{References}

[1] Al-Madi, N.A., Hnaif, A.A. (2021). Optimizing traffic signals in smart cities based on genetic algorithm. Computer Systems Science and Engineering, 40(1), 65-74.

[2] Banharnsakun, A. (2018). Multiple traffic sign detection based on the artificial bee colony method. Evolving Systems, 9(3), 255-264.

[3] Blagojević, A., Kasalica, S., Stević, Ž., Tričković, G., Pavelkić, V. (2021). Evaluation of Safety Degree at Railway Crossings in Order to Achieve Sustainable Traffic Management: A Novel Integrated Fuzzy MCDM Model. Sustainability, 13(2), 1-20.

[4] Brzozowska, A., Bubel, D., Kalinichenko, A. (2019). Analysis of the road traffic management system in the neural network development perspective. EasternEuropean Journal of Enterprise Technologies, 2(3-98), 1624.

[5] Cui, H., Yuan, G., Liu, N., Xu, M., Song, H. (2020). Convolutional neural network for recognizing highway traffic congestion. Journal of Intelligent Transportation Systems: Technology, Planning, and Operations, 24(3), 279-289.

[6] Dell'Orco, M., Başkan, Ö., Marinelli, M. (2014). Artificial bee colony-based algorithm for optimising traffic signal timings. Advances in Intelligent Systems and Computing, 223, 327-337.

[7] Deveci, M., Pamucar, D., Gokasar, I. (2021). Fuzzy Power Heronian function based CoCoSo method for the advantage prioritization of autonomous vehicles in realtime traffic management. Sustainable Cities and Society, 69.

[8] Do, L.N.N., Taherifar, N., Vu H.L. (2019). Survey of neural network-based models for short-term traffic state prediction. WIREs: Data Mining and Knowledge Discovery, 9(1), 1-24.

[9] Elgarej, M., Khalifa, M., Youssfi, M. (2016). Traffic lights optimization with distributed ant colony optimization based on multi-agent system. Networked Systems, 266-279.

[10] Engelbrecht, A. (2007). Computational Intelligence: An Introduction. Wiley, New York.

[11] Gu, X., Li, T., Wang, Y., Zhang, L., Wang, Y. (2018). Traffic fatalities prediction using support vector machine with hybrid particle swarm optimization. Journal of Algorithms and Computational Technology, 12(1), 20-29.

[12] Hanne, T., Dornberger, R. (2017). Computational Intelligence in Logistics and Supply Chain Management. Springer International Publishing Switzerland.

[13] Hu, W., Wang, H., Qiu, Z., Nie, C., Yan, L. (2018). A quantum particle swarm optimization driven urban traffic light scheduling model. Neural Computing and Applications, 29(3), 901-911.

[14] Hu, Y., Liu, K., Zhang, X., Su, L., Ngai, E.W.T., Liu, M. (2015). Application of evolutionary computation for rule 
discovery in stock algorithmic trading: A literature review. Applied Soft Computing Journal, 36, 534-551.

[15] llin, V., Simić, D., Simić, S.D., Simić, S. (2020). Hybrid Genetic Algorithms and Tour Construction and Improvement Algorithms Used for Optimizing the Traveling Salesman Problem. 15th International Conference on Soft Computing Models in Industrial and Environmental Applications, 530-539.

[16] Kannan, S., Dhiman, G., Natarajan, Y., Sharma, A., Mohanty, S.N. (2021). Ubiquitous vehicular ad-hoc network computing using deep neural network with iot-based bat agents for traffic management. Electronics, 10(7), 1-16.

[17] Karaboga, D., Gorkemli, B., Ozturk, C., Karaboga, N. (2014). A comprehensive survey: artificial bee colony (abc) algorithm and applications. Artificial Intelligence Review, 42(1), 21-57.

[18] Khattak, Z.H. Fontaine, M.D. (2020). A Bayesian modeling framework for crash severity effects of active traffic management systems. Accident Analysis and Prevention, $145,1-32$.

[19] Kumar, P.M., Devi G U., Manogaran, G., Sundarasekar, R., Chilamkurti, N., Varatharajan, R. (2018). Ant colony optimization algorithm with Internet of Vehicles for intelligent traffic control system. Computer Networks, 144, 154-162.

[20] Meicler, A., Sanogo, A., Shvai, N., Llanza, A., Hasnat, A. Khata, M., Younes, E.-D., Khalil, A., Lachachi, Y., Nakib, A. (2020). Real time automatic urban traffic management framework based on convolutional neural network under limited resources constraint. Proceedings of the Lecture Notes in Computer Science, 95-106.

[21] Ng, K.K.H., Lee, C.K.M., Zhang, S.Z., Wu, K., Ho, W. (2017). A multiple colonies artificial bee colony algorithm for a capacitated vehicle routing problem and re-routing strategies under time-dependent traffic congestion. Computers and Industrial Engineering, 109, 151-168.

[22] Nguyen, T.-H., Jung, J.J. (2021). Ant colony optimizationbased traffic routing with intersection negotiation for connected vehicles. Applied Soft Computing, 112.

[23] Ospina-Mateus, H., Quintana Jiménez, L.A., Lopez-Valdes, F.J., Berrio Garcia, S., Barrero, L.H., Sana, S.S. (2021). Extraction of decision rules using genetic algorithms and simulated annealing for prediction of severity of traffic accidents by motorcyclists. Journal of Ambient Intelligence and Humanized Computing, 12(11), 10051-10072.

[24] Raskar, C., Nema, S. (2021). Modified Fuzzy-Based Smart Barricade Movement for Traffic Management System. Wireless Personal Communications, 116(4), 3351-3370.

[25] Underwood, R.T. (1990). Traffic management: an introduction. Hartree Publishing.

[26] Wei-jun, P., Jie, R., Run-Dong, W. (2019). Air Traffic Management Process Quality Assessment Model Based on Improved Fuzzy Matter Element Analysis. Journal of Physics: Conference Series, 1187(5), 1-10.
[27] Wilde, H., Knight, V., Gillard, J. (2020). Evolutionary dataset optimisation: learning algorithm quality through evolution. Applied Intelligence, 50, 1172-1191.

[28] Zhang, H.Q., Wang, C.H. (2018). Traffic flow forecasting method based on improved particle swarm optimization algorithm. Advances in Transportation Studies, 3, 109-116. 